\title{
Sound Scattering Properties of Surfaces with Diffusers
}

\author{
Marina $\mathrm{L}_{\text {avrova }}{ }^{1 *}$, and Nikolay $\mathrm{K}$ anev ${ }^{1,2}$ \\ ${ }^{1}$ B auman M oscow State Technical U niversity, 105005, M oscow, Russia \\ ${ }^{2}$ A ndreyev A coustic Institute, 117036, M oscow, Russia
}

\begin{abstract}
This article compares the values of the normal scattering coefficient measured in a model experiment for two types of diffusers placed on a rigid surface. Wooden diffusers of cubic and pyramidal shapes were tested in a scale model of a room with dimensions of $0.7 \times 0.4 \times 0.4 \mathrm{~m}$. Sound decay curves were measured at frequencies of $4 \mathrm{kHz}$ and $8 \mathrm{kHz}$. Two large walls were covered with a porous absorber, on the third, in certain combinations, the investigated diffusers with a characteristic size of $3.5 \mathrm{~cm}$ were placed, the number of which varied from 0 to 29 . The idea of the applied method is that the sound decay curve in a room with a non-diffuse sound field depends significantly from the scattering properties of surfaces. The decay curve was measured with different numbers of the diffusers on the test wall, which made it possible to determine the influence of the shape of the diffusers and their number on the value of the normal scattering coefficient. According to the results of the measurements a high scattering ability of cubic elements was revealed in comparison with pyramidal ones.
\end{abstract}

\section{Introduction}

One of the important acoustic characteristics of a surface is the sound scattering coefficient, which is used to determine the acoustic quality of various rooms: concert halls, sound studios, reverberation chambers. The scattering effect can be achieved by individual elements: columns, statues, decorative elements located in a room, or surfaces with an uneven structure [1-4]. In this paper, we consider flat surfaces with scattering elements located on them.

To determine the scattering properties, there are a number of standardized methods that allow to accurately determine the desired coefficient. However, these techniques require a large chamber and the creation of a diffuse field $[5,6]$. The measurements described in this article were carried out by measuring the scattering coefficient in a nondiffuse sound field [7].

The scattering coefficient is determined from the shape of the attenuation curve, given by an exponential-power function and measured in a rectangular room with an uneven distribution of absorption on its walls. In works [8-12] theoretical substantiation of the new method and the results of experiments on its verification are given. In the present work, this

\footnotetext{
* Corresponding author: marinalavro@ mail.ru
} 
method is developed to measure the scattering coefficient at normal incidence of a sound wave on a scattering surface.

\section{Theoretical basis of the measurement method}

The sound decay curve (the dependence of the logarithmic level of the sound pressure average over the volume of the room on time) depends on the shape and properties of the surfaces of the room and. In the case of a diffuse field it is a straight line, which can be easily described by an angle of inclination or by the reverberation time. In a rectangular room with an uneven distribution of absorption, the decay of sound energy is nonexponential due to the nondiffuseness of the sound field, and depends on the degree of sound scattering. When sound-scattering elements are introduced into the room, sound decays faster and their acoustic properties can be determined from the measured decay curve.

Let us consider a room that has the shape of a rectangular parallelepiped (Fig. 1). Two non-parallel walls are covered with sound-absorbing material, the walls perpendicular to them are test ones, the test samples are placed on them. This creates a non-diffuse sound field necessary for measurements. Finding the scattering coefficient from the measured decay curve is possible only if it differs significantly from the exponential. The absorption and reflection coefficients are used to describe the acoustic properties of surfaces.

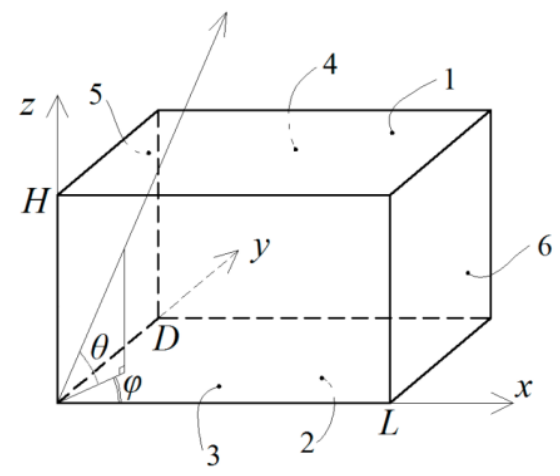

Fig. 1. Rectangular room.

Sound energy in the rectangular room with flat walls decays in accordance with the following law, which is found in [9]

$$
E_{s}(t)=\frac{2}{\pi} \int_{0}^{\frac{\pi}{2}} \int_{0}^{\frac{\pi}{2}} e^{\frac{t}{2} \sum_{i=1}^{6} n_{i} \ln \gamma_{i}} \cos \theta d \theta d \varphi
$$

where $t$ - time;

$i$ - room wall number;

$n_{1,2}=\frac{c}{2 H} \sin \theta, n_{3,4}=\frac{c}{2 D} \cos \theta \sin \varphi, n_{5,6}=\frac{c}{2 L} \cos \theta \cos \varphi$ - number of reflections from the wall with $i$ per time unit number;

$H, L, D$ - the lengths of the sides of the room;

$\gamma_{i}=1-\alpha_{i}$ and $\alpha_{i}$ - the reflection and absorption coefficients of $i$-th wall.

Expression (1) obtained in the geometrical acoustics approximation is valid for frequencies $f>2000 \sqrt{T / V}$ [13], where $f$ is frequency, $\mathrm{Hz}, T$ is the reverberation time, $\mathrm{s}, V$ is the room volume, $\mathrm{m}^{3}$. 
Let us set the acoustic impedance $\mathrm{Z}$ to walls 1 and 3, then their reflection coefficients are found as follows [14]

$$
\gamma_{1}(\theta, \varphi)=\left|\frac{Z \sin \theta-1}{Z \sin \theta+1}\right|^{2}, \quad \gamma_{3}(\theta, \varphi)=\left|\frac{Z \cos \theta \sin \varphi-1}{Z \cos \theta \sin \varphi+1}\right|^{2}
$$

Other walls are fully reflective, i.e. $\gamma_{2}=\gamma_{4}=\gamma_{5}=\gamma_{6}=1$. After substituting the coefficients (2) into (1), the decay law takes the form

$$
E_{S}(t) \sim 1 / t
$$

If scattering elements are applied to the wall 6 , hereinafter referred to as test, not parallel to the absorbing walls, then in accordance with the law of conservation of energy its coefficient of specular reflection $\gamma_{6}(\theta, \varphi)=1-\delta_{6}(\theta, \varphi)$, where $\delta_{6}$ is the scattering coefficient of the test wall, since the absorption coefficient $\alpha_{6}=0$.

Let us take into account the scattering of sound in (1) and determine the decay law for the room with two absorbing walls and one test one under the condition $t>>\frac{L}{c}, \frac{D}{c}, \frac{H}{c}$

$$
E_{S}(t) \approx \frac{\sqrt{H D}}{4 c t \operatorname{Re} Z} e^{-\frac{c t}{2 L} \delta_{6}(0,0)}
$$

Thus, the decay law depends on the normal scattering coefficient of the wall with the number $\mathrm{i}=6$, and the value of this coefficient can be determined from the measured decay curve by approximating it with function (4).

The obtained result has a simple physical interpretation. The initial distribution of the energy transferred along the beams at the angles $\theta$ and $\varphi$ is uniform. First of all, the energy of rays incident on the absorbing wall at large angles is absorbed. The sound field becomes anisotropic in the time required for the sound to travel a distance equal to several free path lengths. The rays propagating along the absorbing walls, i.e. normal to the scattering wall, lose their energy slowly. This is due to the fact that, firstly, these rays are rarely reflected from the absorbing walls, and secondly, because the absorption coefficient for grazing incidence on the impedance walls is close to zero. The decay rate of the energy of these rays depends on the scattering coefficient of the test wall: the higher it is, the more energy is dissipated and falls on the absorbing walls not at grazing angles. The scattered energy is rapidly absorbed, which makes the influence of absorption and scattering of the wall equivalent to the law of sound decay [8].

Full sound energy in the room is determined by the energy specular reflection (4) and the scattered energy. Let us estimate the latter as follows. There is only one wall in the room with a non-zero scattering coefficient $\delta_{6}$. By the time moment the wave, the direction of which is characterized by the angles $\varphi$ and $\theta$, experiences $N=t_{0} \sum_{i=1}^{6} n_{i}(\theta, \varphi)$ mirror reflections. The energy of specular reflections before the last reflection is determined by the first $N-1$ reflections. If the N-reflection occurs from the wall $i=6$, then the energy dissipated by the $N$-th reflection is

$$
\delta_{6} \gamma_{6}^{\frac{t_{0}}{2} n_{6}(\theta, \varphi)-1} \prod_{i \neq 6} \gamma_{i}^{\frac{t_{0}}{2} n_{i}(\theta, \varphi)}=\frac{\delta_{6}}{\gamma_{6}} \prod_{i} \gamma_{i}^{\frac{t_{0}}{2} n_{i}(\theta, \varphi)}=\frac{\delta_{6}}{\gamma_{6}} e^{\frac{t_{0}}{2} \sum_{i} n_{i}(\theta, \varphi) \ln \gamma_{i}} .
$$


During the time interval from $t_{0}$ to $t_{0}+d t_{0}$ there are $n_{6} d t_{0}$ reflections from the diffusing wall. To obtain the scattered energy during this interval, we integrate over the angle

$$
d E_{d}=\frac{d t_{0}}{4 \pi} \int_{0}^{2 \pi} \int_{-\frac{\pi}{2}}^{\frac{\pi}{2}} n_{6}(\theta, \varphi) \frac{\delta_{6}(\theta, \varphi)}{\gamma_{6}(\theta, \varphi)} e^{\frac{t_{0}}{2} \sum_{i} n_{i}(\theta, \varphi) \ln \gamma_{i}} \cos \theta d \theta d \varphi .
$$

At time $t_{0}$ the dissipated energy is equal to $d E_{d}$. It decays according to (1), therefore, by the moment of time $t$ it is $E_{s}\left(t-t_{0}\right) d E_{d}$. To obtain the dissipated energy in the room by the time moment, we have to integrate (6) in time from the beginning of the reverberation process

$$
E_{d}=\frac{1}{4 \pi} \int_{0}^{t} d t_{0} E_{s}\left(t-t_{0}\right) \int_{0}^{2 \pi} \int_{-\frac{\pi}{2}}^{\frac{\pi}{2}} n_{6}(\theta, \varphi) \frac{\delta_{6}(\theta, \varphi)}{\gamma_{6}(\theta, \varphi)} e^{\frac{t_{0}}{2} \sum_{i} n_{i}(\theta, \varphi) \ln \gamma_{i}} \cos \theta d \theta d \varphi .
$$

Substituting (4) into (7), we find an approximate expression for the scattered energy

$$
E_{d}(t) \approx \frac{\pi^{2}}{64} \frac{\delta_{\perp}}{1-\delta_{\perp}} \frac{H D}{c t L \operatorname{Re} Z} e^{-\frac{c t}{2 L} \delta_{\perp}}
$$

where $\delta_{\perp}=\delta_{6}(0,0)$.

The energy of specular reflections (4) and the scattered energy (8) depend on time in the same way, so the dependence of the total sound energy in the room $E=E_{s}+E_{d}$ on time has the form

$$
E(t) \approx \frac{a}{t} e^{-b t}
$$

Thus, the measured decay curve is approximated by dependence (9), the coefficients a and $b$ are determined, the scattering coefficient is found from the expression

$$
\delta_{\perp}=\frac{2 L}{c} b .
$$

Note that, in deriving (8), only single scattering of sound has been considered. After a while, the rays scattered at each reflection will fall again on the diffuse wall, the scattered energy will also contribute to the total sound energy in the room, which has not been taken into account in (8). The value of this energy can also be estimated as the energy of single scattering (7); it is obvious that it will be proportional $\delta_{\perp}^{2}$. In a similar way, the energy of multiple scattering can be introduced, and it will be proportional $\delta_{\perp}^{m}$, where $m$ - the number of reflections from the scattering wall.

Energy $E_{d}$ taking into account all diffuse reflections decays according to the law (9) as well, so it could be concluded that sound decay in the room occurs according to the same law for any values of the scattering coefficient. However, (9) has been obtained taking into account dependence (4), which is valid for $t>>\frac{L}{c}$, i.e. it is assumed that the scattering on the wall is small, so the scattered energy does not have time to be quickly absorbed, and its decay occurs according to law (9). If a significant fraction of the energy is dissipated upon reflection from the diffuse wall, then, firstly, the energy of specular reflections $E_{s}$ decreases rather 
quickly, and secondly, the dissipated energy decays according to law (1) at $t \sim \frac{L}{c}$, which differs from the approximate decay law (4). This paper does not consider the case of large scattering coefficients, but in the limiting case $\delta_{\perp} \rightarrow 1$ the decay law should go over to the usual Sabin's law [15].

\section{Model experiment}

The proposed method for measuring the scattering coefficient of sound at normal incidence has been verified in a model experiment. In a rectangular room (Fig. 2) with dimensions of $0.7 \times 0.4 \times 0.4 \mathrm{~m}$, two non-parallel long walls are covered with sound-absorbing material. One of the walls measuring $0.4 \times 0.4 \mathrm{~m}$ is designed to accommodate the tested sound diffusers. In one of the corners of the room, there is a sound source with a sufficiently wide directivity pattern to consider the sound field isotropic at the initial moment of time. The decay curve is measured with an omnidirectional microphone located near the test wall.

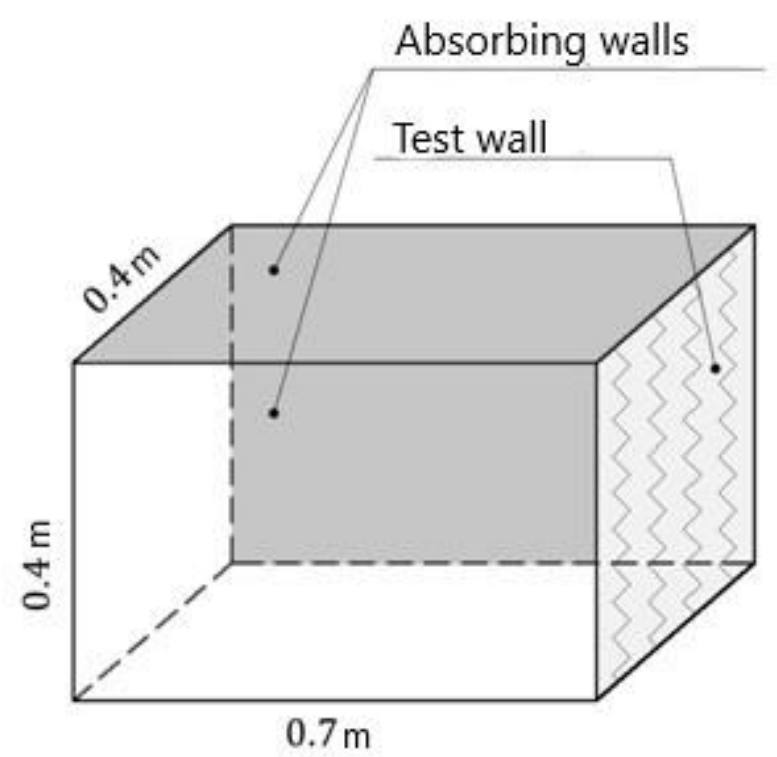

Fig. 2. Room model.

The approximation of the measured curve by dependence (9) was carried out on a time interval from $t_{1}$ to $t_{2}$. The lower bound $t_{1}$ is taken from the ratio $t>>\frac{L}{c}=2 \mathrm{~ms}$. The $t_{2}$ value corresponds to the point in time when the decay curve value on $10 \mathrm{~dB}$ higher than the background noise value. Curve coefficients are fitted by the least squares method. A detailed description of the processing of impulse responses is presented in articles [16-18].

As the diffuse element used in the experiment wooden cubes and pyramids with a characteristic dimension of $3.5 \mathrm{~cm}$. The elements stuck in a specific pattern to (Fig.3) test wall surface, their number varied from 2 to 29 . 


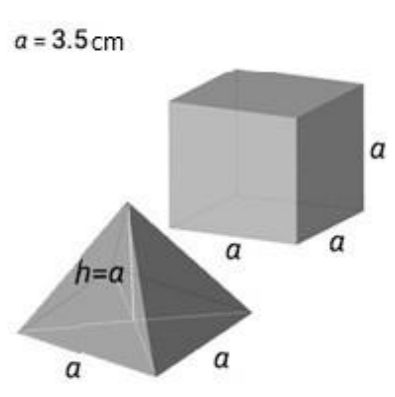

(a)

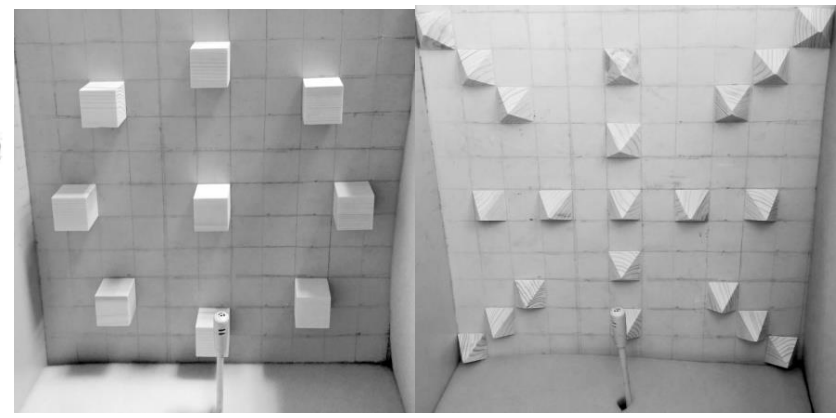

(b)

(c)

Fig. 3. Dimensions (a) and photo of the location of the scatterers (b, c).

As noted above, the absorption and scattering coefficients have the same effect on the sound decay curve. Therefore, it is impossible to unambiguously single out the impact of each coefficient separately. In order to solve this problem, it is necessary to find the sound absorption coefficient of the test elements $\alpha$ by an alternative method [19] and determine the difference.

\section{$3 \quad$ Measurement results}

Let us consider the obtained dependences of the sum $\delta_{n}^{\prime}=\alpha_{n}+\delta_{n}$ of the normal scattering coefficient and the absorption coefficient depending on the number of elements $n$ for the range 0-29 (Fig. 4). The elements were located on the surface with the condition of uniform coverage of the test wall.

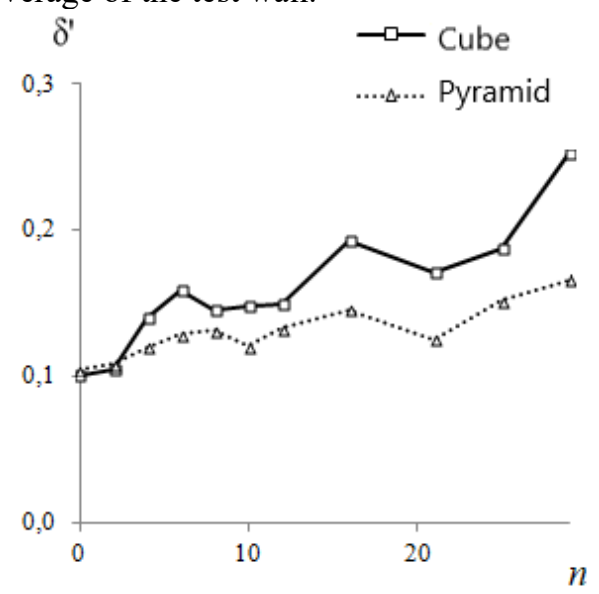

(a)

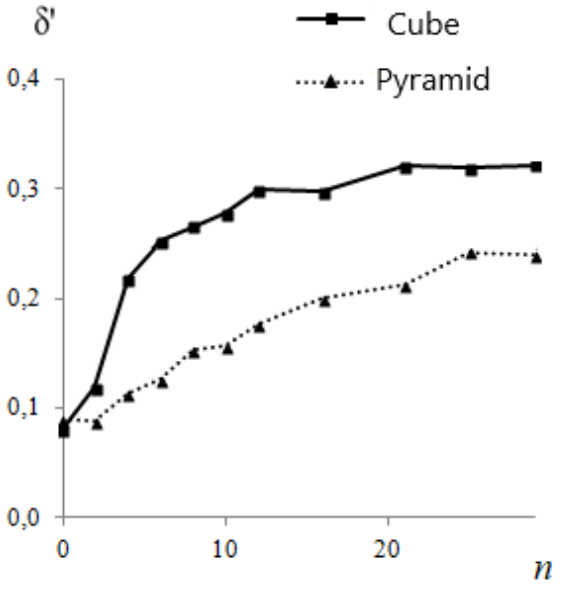

(b)

Fig. 4. The results of measuring the total absorption and scattering coefficient $\delta$ ( of the test wall at 4 $\mathrm{kHz}$ (a) and $8 \mathrm{kHz}$, depending on the number of scatterers $\mathrm{n}$.

With a small number of elements, the main contribution to the value of the coefficient $\delta_{n}^{\prime}$ is made precisely by the absorption of sound by the surface of the test walls. The wall absorption coefficient can be taken as the value of the empty wall coefficient $\alpha=\delta^{\prime}{ }_{0}=0.1$.

The empirically obtained data clearly show the dependence of the growth of the normal scattering coefficient on the number of the diffusers on the test wall. Moreover, the growth of the total coefficient of scattering and absorption of pyramidal diffusers occurs gradually 
without sharp jumps at both $4 \mathrm{kHz}$ and $8 \mathrm{kHz}$. Maximum value at 29 elements at $4 \mathrm{kHz}: \delta_{29}^{\prime}=$ 0.166 at $8 \mathrm{kHz}: \delta_{29}^{\prime}=0.240$.

For cubic diffusers at $8 \mathrm{kHz}$, growth occurs most intensively in the range of 2-6 elements. Further, with an amount of 10-29, the coefficient hardly changes. Maximum value at $4 \mathrm{kHz}$ : $\delta_{29}^{\prime}=0.252$, at $8 \mathrm{kHz}: \delta_{29}^{\prime}=0.322$.

Such diffusers with a characteristic size of $3.5 \mathrm{~cm}$ scatter sound at a frequency of $8 \mathrm{kHz}$ due to the fact that the size is close to the length of the sound wave

$$
\lambda=\frac{c}{f}=\frac{340 \mathrm{~m} / \mathrm{c}}{8000 \mathrm{c}^{-1}}=4.2 \mathrm{~cm}
$$

\section{$4 \quad$ Estimated calculation of scattering coefficient}

For a qualitative explanation of the experimental results obtained, let us consider the problem of the scattering of a sound wave on a plane with periodic inhomogeneities in the twodimensional case, where the surface consists of simple geometric figures periodically located on the plane. Let us consider squares and triangles as figures, since in this work cubic and pyramidal elements are investigated.

Figure 5 shows a plane with rectangular scatterers of sizes $a$ and $d$ with period $L$.

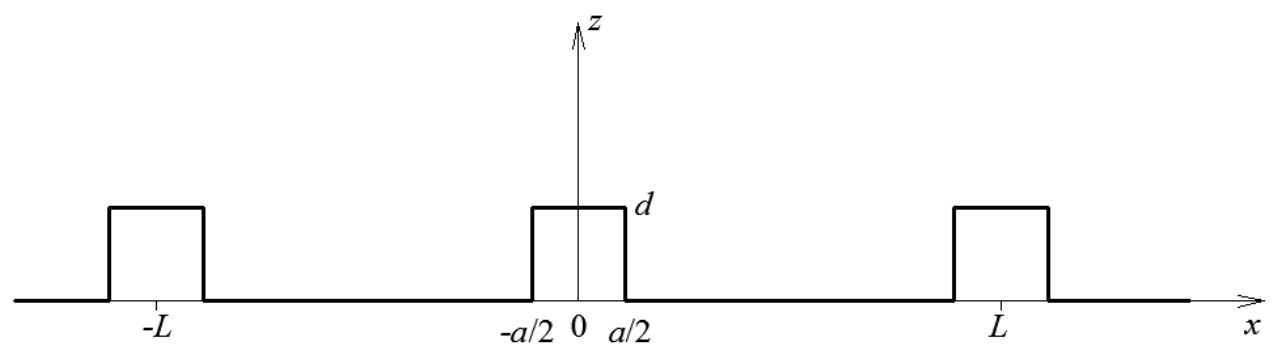

Fig. 5. Section of the surface with rectangular diffusers.

The surface profile $z_{1}(x)$ can be represented as a Fourier series, i.e. as a superposition of sinusoidal profile irregularities:

$$
z_{1}(x)=\sum_{n=1}^{\infty} h_{n} \cos \left(\xi_{n} x\right),
$$

where $h_{n}=\frac{2 d}{\pi n} \sin \left(\pi n \frac{a}{L}\right)-$ the height of the sinusoidal unevenness above the surface $z=0$; $\xi_{n}=2 \pi \frac{n}{L}$ - dimensionless parameter characterizing the period of inhomogeneity.

The scattering of sound on a slightly rough surface can be found by the method of small perturbations [14], which has two conditions of applicability: the smallness of the height of the roughness in comparison with the wavelength and the slope of the surface. For each component of expansion (12), these conditions have the form

$$
\begin{aligned}
& k h_{n}<<1, \\
& \xi_{n} h_{n}<<1,
\end{aligned}
$$

where $k=\omega / c, \omega$ is the frequency, $c$ is the speed of sound. 
Let the dimensions of the inhomogeneities be small compared to the period $a, d<<L$, then for the first terms of the sum (12), the numbers $n$ of which satisfy the condition $\pi n a / L<<1$, the height of the inhomogeneity is $h_{n}=2 \frac{a d}{L}$. For large values of $n$ the height of the inhomogeneity satisfies the condition $h_{n}<\frac{2 d}{\pi n}$. Thus, the condition of applicability (13) is reduced to the condition $2 k \frac{a d}{L}<<1$.

Let us estimate the second condition of applicability

$$
\xi_{n} h_{n}=\frac{4 d}{L} \sin \left(\pi n \frac{a}{L}\right)<\frac{4 d}{L} .
$$

It follows from (15) that the maximum angle of inclination of any component of expansion (12) does not depend on its number, and condition (14) is reduced to the ratio of the depth and period of inhomogeneity $\frac{4 d}{L}<<1$.

Consider the normal incidence of a plane sound wave of unit amplitude on a scattering surface

$$
p_{0}=e^{-i k z}
$$

The field scattered on a sinusoidal surface numbered is written in the form [14]

$$
\begin{gathered}
p_{n}=A_{n}\left(e^{i \xi_{n} x+i \sqrt{k^{2}-\xi_{n}^{2}} z}+e^{-i \xi_{n} x+i \sqrt{k^{2}-\xi_{n}^{2}} z}\right) \\
A_{n}=-i \frac{k h_{n}}{\sqrt{1-\left(\frac{\xi_{n}}{k}\right)^{2}}} .
\end{gathered}
$$

The scattered field represents two plane waves leaving the plane at an angle $\theta_{n}$, that satisfies the condition $\operatorname{tg} \theta_{n}=\xi_{n} / k$. The wave is homogeneous, i.e. running along the direction given by the angle $\theta_{n}$, under the following condition $\xi_{n}<k$.

If, on the contrary $\left(\xi_{n}>k\right)$ the scattered waves are inhomogeneous, i.e. exponentially decaying along the $z$ axis. Such waves do not carry sound energy away from the scattering surface. We are primarily interested in scattered sound energy, i.e. energy leaving the surface in directions other than normal. Let $N$ - the maximum value of $n$, that satisfies the condition $\xi_{n}>k$. The energy scattered by the surface $z_{1}(x)$ is determined by the sum of the energies of all scattered homogeneous waves

$$
\delta=2 \sum_{n=1}^{N}\left|A_{n}\right|^{2} .
$$

Since the incident wave energy is 1 , the value $\delta$ is the surface scattering coefficient. Let us calculate the characteristics of the scattered field depending on the size of the scattered $a=d=3 \mathrm{~cm}$ (Table 1$)$.

Let's carry out similar calculations for a triangular section (Fig. 6). The height of the sinusoidal roughness of a given surface is described by the formula 


$$
h_{n}=\frac{4 d L}{a(\pi n)^{2}} \sin ^{2} \frac{\pi n a}{2 L} .
$$

Table 1. Characteristics of the scattered field of cubic elements at $4 \mathrm{kHz}$.

\begin{tabular}{|l|c|c|c|c|c|c|}
\hline Calculated parameter & \multirow{2}{*}{ Formula } & \multicolumn{5}{|c|}{ n } \\
\cline { 3 - 7 } & & $\mathbf{1}$ & $\mathbf{2}$ & $\mathbf{3}$ & $\mathbf{4}$ & $\mathbf{5}$ \\
\hline Roughness height & $k_{n}$ & 0.221 & 0.218 & 0.214 & 0.207 & 0.200 \\
\hline Maximum surface slope & $\xi_{n} h_{n}$ & 0.031 & 0.062 & 0.091 & 0.118 & 0.141 \\
\hline Scattering angle & $\theta_{n}$ & 0.141 & 0.276 & 0.402 & 0.516 & 0.616 \\
\hline Scattered wave amplitude & $\left|A_{n}\right|$ & 0.223 & 0.227 & 0.236 & 0.252 & 0.283 \\
\hline Scattered wave energy & $2\left|A_{n}\right|^{2}$ & 0.1 & 0.103 & 0.111 & 0.127 & 0.16 \\
\hline Scattering coefficient & $\delta$ & & & 0.601 & & \\
\hline
\end{tabular}

The dimensions of the scatterer are assumed to be the same $a=d=3 \mathrm{~cm}$. The period is selected so that the conditions of applicability (13) and (14) are satisfied. In these calculations, the period is $L=20 a=60 \mathrm{~cm}$.

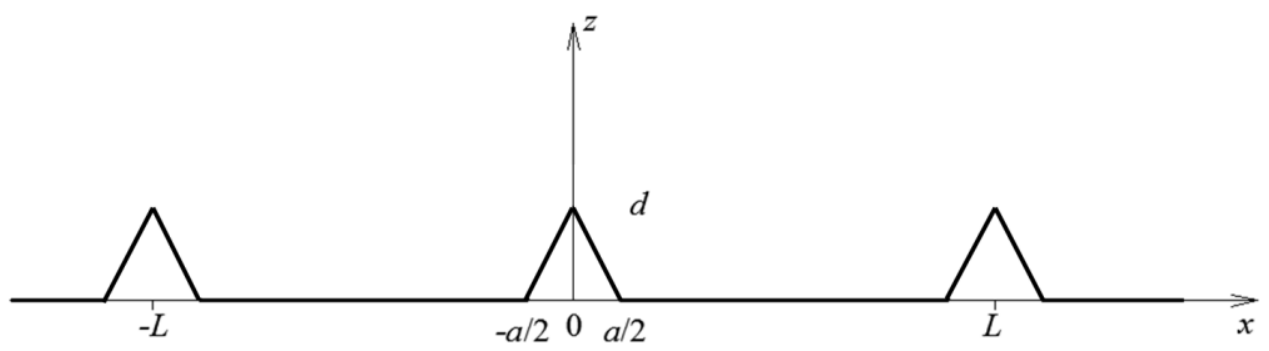

Fig. 6. Section of the surface with pyramidal diffusers.

Below are the calculations of the scattering coefficient (Table 2) for a triangular section with the number of scatterers on the plane not exceeding 5 .

The theory on the estimation of the numerical value of the scattering coefficient is confirmed by the experiment: the scattering coefficient for cubic elements is much higher than the coefficient for pyramidal ones. 
Table 2. Characteristics of the scattered field of pyramidal elements at $4 \mathrm{kHz}$

\begin{tabular}{|l|c|c|c|c|c|c|}
\hline \multirow{2}{*}{ Calculated parameter } & \multirow{2}{*}{ Formula } & \multicolumn{5}{|c|}{$n$} \\
\cline { 3 - 7 } & & $\mathbf{1}$ & $\mathbf{2}$ & $\mathbf{3}$ & $\mathbf{4}$ & $\mathbf{5}$ \\
\hline Roughness height & $k_{n}$ & 0.111 & 0.110 & 0.109 & 0.107 & 0.105 \\
\hline Maximum surface slope & $\xi_{n} h_{n}$ & 0.016 & 0.031 & 0.046 & 0.061 & 0.075 \\
\hline Scattering angle & $\theta_{n}$ & 0.141 & 0.276 & 0.402 & 0.516 & 0.616 \\
\hline Scattered wave amplitude & $\left|A_{n}\right|$ & 0.112 & 0.115 & 0.120 & 0.130 & 0.149 \\
\hline Scattered wave energy & $2\left|A_{n}\right|^{2}$ & 0.025 & 0.026 & 0.029 & 0.034 & 0.045 \\
\hline Dissipation factor & $\delta$ & & & & & \\
\hline
\end{tabular}

In the case of cubic scatterers for the first terms of (12), whose numbers $n$ satisfy the condition $\pi n a / 2 \ll 1$, the height of the inhomogeneity is $h_{n}=2 \frac{a d}{L}$. Then the value of the scattered wave amplitude

$$
\begin{gathered}
A_{n} \approx k h_{n}=k \frac{2 d}{\pi n} \sin \frac{\pi n a}{L}=2 k \frac{a d}{L}, \\
A_{n} \approx 2 k \frac{S}{L} .
\end{gathered}
$$

where $S=a d$ - the sectional area of the element.

Taking into account that $\pi n a / 2 L \ll 1$ for pyramidal diffusers the height of the inhomogeneity is given by

$$
h_{n}=\frac{4 d L}{a(\pi n)^{2}} \sin ^{2} \frac{\pi n a}{2 L}=\frac{4 d L}{a(\pi n)^{2}}\left(\frac{\pi n a}{2 L}\right)^{2}=\frac{d a}{L} .
$$

Then the value of the scattered wave amplitude

$$
A_{n} \approx k h_{n}=k \frac{d a}{L}=k \frac{S}{L}
$$

From (22) and (24) it follows that the amplitude of the scattered wave is proportional to the volume of the scattering element. In the two-dimensional case it is the area $S$ for rectangular scatterers and $S / 2$ for triangular scatterers. A similar pattern can be expected in the three-dimensional case. 


\section{Conclusion}

As a result of comparative measurements of the scattering coefficient of cubic and pyramidal diffusers at normal incidence of a sound wave, it was found that cubic diffusers are much more effective than pyramidal ones if the distance between the elements is much larger than their characteristic size. This fact can be useful in developing acoustic solutions for different rooms, in particular for multipurpose halls. In the design manual for such halls [20], the choice of elements is based only on their geometric dimensions (Fig. 7).

Fig. 7 shows recommendations for choosing the size of sound-scattering elements according to [20,21]. It follows that for sound scattering in a certain frequency range, it is necessary to choose the values of the element width $b$, the profiling depth $d$ and the period $\mathrm{g}$, but the difference between the element shapes is not indicated. Therefore, we can assume that rectangular, triangular and semi-cylindrical elements have the same scattering properties.

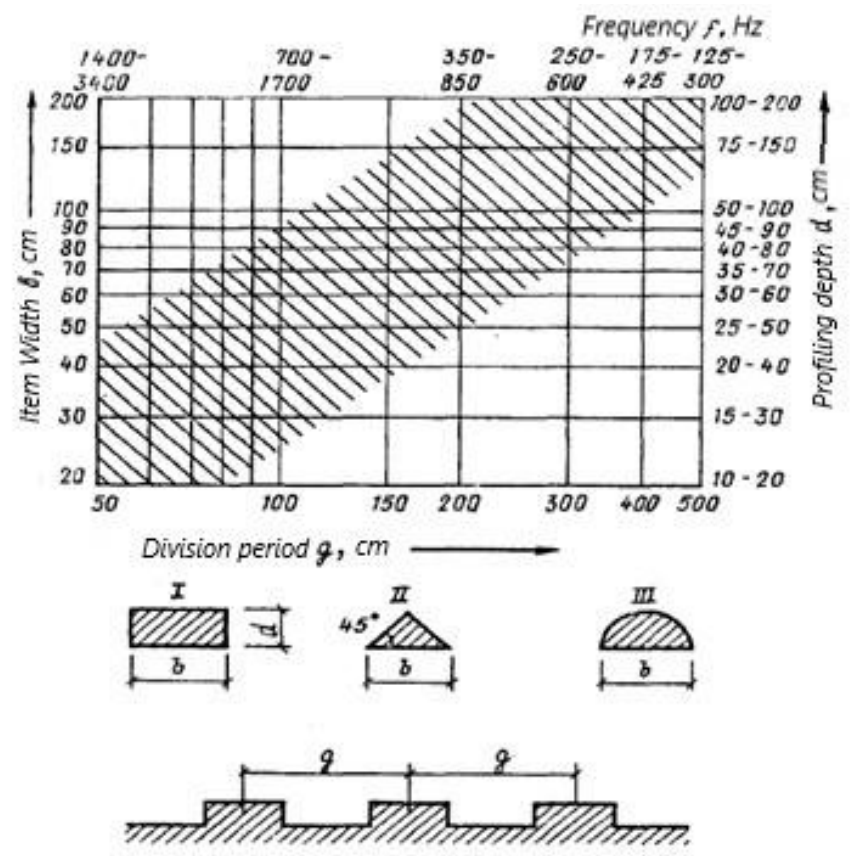

Fig. 7. The approximate dimensions of the periodic divisions, providing the scattering of the reflected sound of different frequencies [20].

According to the results obtained in this work, when choosing solutions for sound scattering using relief surfaces, it is necessary to take into account the shape of the scattering elements [22], which would be useful to indicate in the recommendations [20].

\section{References}

1. T.J. Cox, B.I.L. Dalenback, P. D \& ntonio, J.J . E mbrechts, J.Y . Jeon, E. M ommert, M. Vorländer, J.Acta Acust united Ac, 1, 1-15 (1992)

2. T.J. Cox, P. D A ntonio, Acoustic Absorbers and Diffusers: Theory Design and Application, Spon Press, London (2004)

3. W. Shuping, T. Jiancheng, Q. X iaojun, C. J ianchun, J. of Sound and V ibration, 1-14 (2018) 
4. M. Vorländer, E. Mommertz, Appl. Acoust. 187-199 (2000)

5. ISO 17497-1-2011. A coustics - Sound-scattering properties of surfaces - Part 1: $M$ easurement of the random-incidence scattering coefficient in a reverberation room.

6. ISO 17497-2:2012. A coustics - Sound-scattering properties of surfaces - Part 2: $M$ easurement of the directional diffusion coefficient in a free field.

7. T. Hanyu, K. Hoshi, T. Nakakita, Method for measuring sound scattering coefficients of walls and diffusers by using a non-diffuse sound field with unevenly-distributed sound absorption, in Proceedings of the 45th I nternational Congress INTER-NOISE, 1022-1030, Hamburg, Germany (2016).

8. M .R. Hodgson, R.J . Orlowski, J. of Sound and V ibration, 113 (1), 29-46 (1987)

9. N.G. Kanev, A coust. Phys. 58, No. 5, 656-662 (2012)

10. N.G. Kanev, A coust. Phys. 63, No. 6, 731-736 (2017)

11. N.G. Kanev, Sound Decay in a Rectangular Room with Impedance Walls, in Proceedings of the INTER N OISE, 2367-2378, Hong K ong, China (2017)

12. N.G. Kanev, On Measuring the Scattering Coefficient in a Nondiffuse Sound Field, in Proceedings of the Forum A custicum, 679-684, A alborg, Denmark (2011)

13. H. K uttruff, Room Acoustics, Spon Press, London, 2000.

14. M.A. Isakovich, General acoustics. Tutorial. Publishing House Science, M oscow (1973)

15. W.C. Sabine, Collected Papers on Acoustics, Cambridge, M A (1922)

16. M.A. Lavrova, N.G. Kanev, Scattering of sound waves by cubic elements in a model experiment, in Proceedings of the Third A II-R ussian conference of young scientists and specialists "A coustics of the environment", 163-170, M oscow (2018)

17. P.A. Trushina, On the possibility of measuring the anisotropy of the scattering coefficient of sound in a non-diffuse field, in Proceedings of the Second All-R ussian conference of young scientists and specialists "A coustics of the environment", 195203, M oscow (2017)

18. P.A. Trushina, N.G. K anev, M emoirs of the Faculty of Physics, Lomonosov M oscow State University, 5, 1751112 (2017)

19. GOST 31704-2011. Sound-absorbing materials. A method for measuring sound absorption in a reverberation chamber.

20. Guidance on acoustic design of multi-purpose halls of medium capacity, R esearch Institute of Construction Physics (RICP) of the USSR Gosstroy, M oscow (1981)

21. L.I. M akrinenko, A coust. Soc. A m., 172 (1994)

22. A.A. Strokin, Modelling of noise reduction using reverberation fields of echoic rooms, in Proceedings of the A IP Conference, 2195, N 0. 1, 020072 (2019) 